\title{
Inhomogeneous chemical enrichment in the Galactic Halo
}

\author{
Chiaki Kobayashi \\ Centre for Astrophysics Research, Science and Technology Research Institute, University of \\ Hertfordshire, Hertfordshire, AL10 9AB, UK; email: c.kobayashi@herts.ac.uk
}

\begin{abstract}
In a galaxy, chemical enrichment takes place in an inhomogeneous fashion, and the Galactic Halo is one of the places where the inhomogeneous effects are imprinted and can be constrained from observations. I show this using my chemodynamical simulations of Milky Way type galaxies. The scatter in the elemental abundances originate from radial migration, merging/accretion of satellite galaxies, local variation of star formation and chemical enrichment, and intrinsic variation of nucleosynthesis yields. In the simulated galaxies, there is no strong age-metallicity relation. This means that the most metal-poor stars are not always the oldest stars, and can be formed in chemically unevolved clouds at later times. The long-lifetime sources of chemical enrichment such as asymptotic giant branch stars or neutron star mergers can contribute at low metallicities. The intrinsic variation of yields are important in the early Universe or metal-poor systems such as in the Galactic halo. The carbon enhancement of extremely metal-poor (EMP) stars can be best explained by faint supernovae, the low $[\alpha / \mathrm{Fe}]$ ratios in some EMP stars naturally arise from low-mass $\left(\sim 13-15 M_{\odot}\right)$ supernovae, and finally, the $[\alpha / \mathrm{Fe}]$ knee in dwarf spheroidal galaxies can be produced by subclasses of Type Ia supernovae such as SN 2002cx-like objects and sub-Chandrasekhar mass explosions.
\end{abstract}

Keywords. Galaxy: abundances, Galaxy: evolution, Galaxy: halo, supernovae: general

\section{Introduction}

Different elements and isotopes are produced from stars on different timescales. Elemental and isotopic abundance ratios of interstellar medium (ISM) evolve as a function of time in a galaxy. Therefore, stars are fossils that retain information on the formation and evolutionary history of the galaxy. This approach is called galactic archaeology. Nucleosynthesis yields have recently been updated and a complete yield table is provided for $1-300 M_{\odot}$ (Nomoto, Kobayashi \& Tominaga 2013, hereafter NKT13). Galactic chemical evolution models (Kobayashi et al. 2006, Kobayashi et al. 2011a) are in excellent agreement with observations except for some minor elements. The $\nu$-process plays an essential role in the production of $\mathrm{F}$ (Kobayashi et al. 2011b), while multi-dimensional calculation is necessary for $\mathrm{Ti}$ (Kobayashi et al. 2006).

Galactic chemical evolution has been studied with so-called one-zone models (also called monolithic models in the cosmological context) for many years. In one-zone models, it is assumed that the interstellar medium (ISM) of the region under consideration is well mixed instantaneously and has a uniform chemical composition. In reality, the ISM is not well mixed and more-realistic models have been proposed. Stochastic models can involve inhomogeneous mixing statistically. Hierarchical (semi-analytic) models include cosmological mass accretion (see NKT13 for the references).

Thanks to the development of high-performance computers and numerical techniques, it became possible to calculate chemical enrichment combined with 3D hydrodynamical simulations, which are called chemodynamical simulations (e.g., Kobayashi \& Nakasato 2011, hereafter KN11, and references therein). With such chemodynamical simulations, positions, kinematics, ages, metallicities, and elemental abundances of star particles are 
obtained. Thus, the scatter of observational data and the effect of inhomogeneous mixing may be studied. These are the predictions that can be, and should be, compared with future observations with high-resolution multi-object spectrographs such as APOGEE, HERMES, and GAIA-ESO surveys.

\section{Dependence on locations in chemodynamical simulations}

We use a hydrodynamical code that includes relevant physical processes such as radiative cooling, star formation, supernova feedback, and chemical enrichment from corecollapse supernovae (SNe II and hypernovae), Type Ia supernovae (SNe Ia), and asymptotic giant branch (AGB) stars. The progenitor model of SNe Ia is based on the single degenerate scenario with the metallicity effects of white dwarf (WD) winds, and the lifetime distribution functions are calculated as in Kobayashi \& Nomoto (2009). See Kobayashi (2004), Kobayashi, Springel \& White (2007), and KN11 for the details. We use $\Lambda$ CDM initial conditions, so that any galaxy forms through the successive merging of subgalaxies with various masses. The initial condition is chosen in order to avoid major mergers at $z \lesssim 2$, which is necessary to retain the disk structure. In this simulated galaxy, the bulge is formed by the initial starburst that is induced by the assembly of gas-rich sub-galaxies. Because of the angular momentum, the gas accretes onto the plane forming a rotationally supported disk that grows from inside out. In the disk, star formation takes place with a longer timescale, which is maintained not by the slow gas accretion, but by the self-regulation due to supernova feedback. Many satellite galaxies successively come in and disrupt, and half of thick disk stars have formed in merging galaxies.

In a real galaxy, the star formation history is not so simple and in particular the interstellar medium (ISM) is not homogeneous at any time, which is different from one-zone chemical evolution models. The effects of inhomogeneous enrichment can be summarized as follows. (I) There is a local variation in star formation and metal flow by the inflow and outflow of the ISM. (II) Heavy elements are distributed via stellar winds and supernovae, and the elemental abundance ratios depends on the metallicity and mass of progenitor stars. (III) The ISM may be mixed before the next star formation by other effects such as diffusion and turbulence. (IV) There is a mixing of stars due to dynamical effects such as merging and migration.

In chemodynamical simulations (KN11; Kobayashi 2014, hereafter K14), hydrodynamics and chemical enrichment are solved self-consistently throughout the galaxy formation. Star formation and chemical enrichment depend on the local density. With our feedback scheme, star particles obtain heavy elements from the gas particles from which the stars form. Gas particles obtain heavy elements only when they pass through within a feedback radius of dying star particles. The mass and metallicity dependencies on nucleosynthesis yields are included by looking at the age and metallicity of progenitor star particles, although the energy dependence has not been included. Because it is not possible to resolve supernova ejecta, the size of feedback region, or the number of gas particle that receive feedback, is given by a parameter. This will account for some of gas-phase mixing. Therefore, (I), (II), (IV) are naturally included but (III) is probably not enough.

Because star formation and chemical enrichment histories depend on locations inside the galaxy, elemental abundances also depend on locations. Figure 1 shows $[\mathrm{O} / \mathrm{Fe}]-[\mathrm{Fe} / \mathrm{H}]$ relations as a function of Galactocentric radius and latitude. Because of the delayed enrichment of SNe Ia, which produce more iron-peak elements than $\alpha$ elements $(\mathrm{O}, \mathrm{Mg}$, $\mathrm{Si}, \mathrm{S}$, and $\mathrm{Ca})$, there is a plateau at $[\mathrm{Fe} / \mathrm{H}] \sim-1$, and then a decreasing trend with $[\mathrm{Fe} / \mathrm{H}]$. The $\alpha$-enhanced population is more prominent at the center of galaxy and at higher latitudes, and very similar figures are obtained with APOGEE and RAVE. 


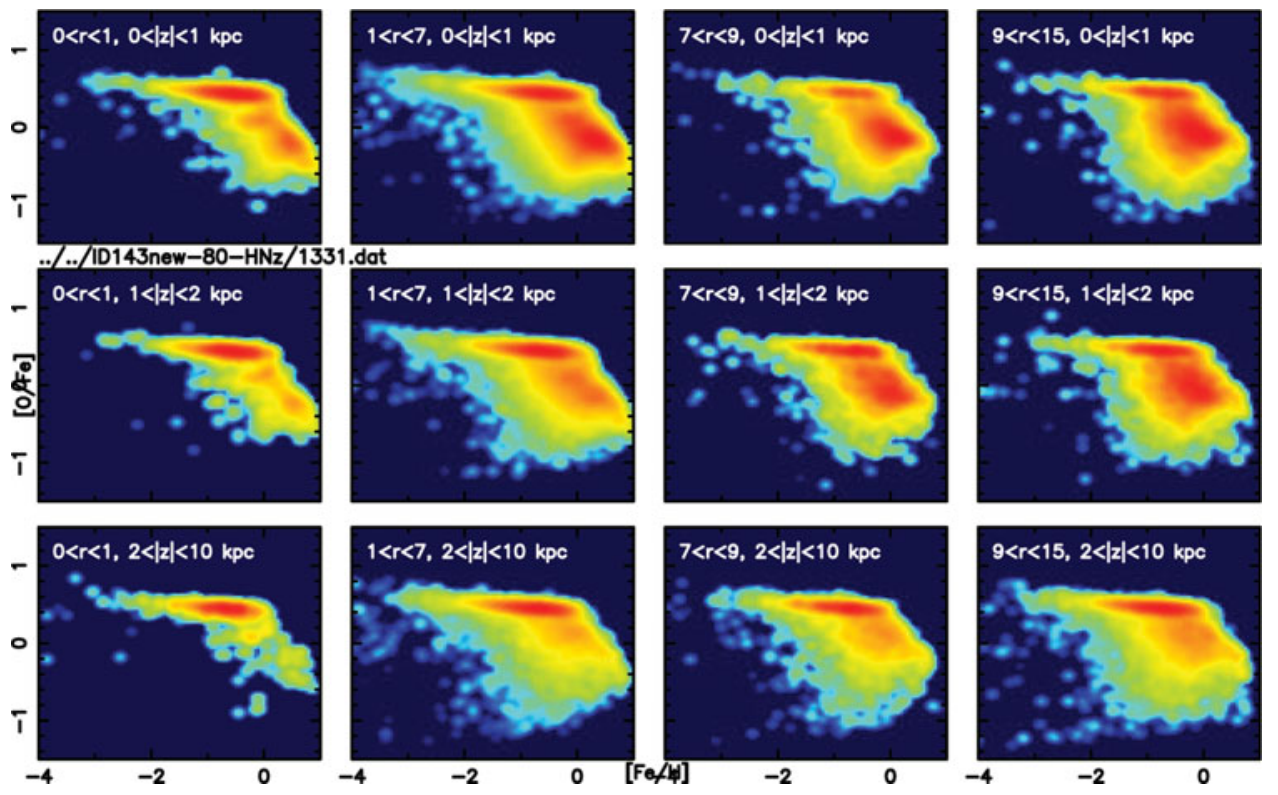

Figure 1. $[\mathrm{O} / \mathrm{Fe}]-[\mathrm{Fe} / \mathrm{H}]$ relations as a function of Galactocentric radius $(r<1,1-7,7-9,9-15$ $\mathrm{kpc})$ and latitude $(z<1,1-2,2-10 \mathrm{kpc})$. The contours show the frequency distribution of stars in the simulated galaxies, where red is for the highest frequency.

In the solar neighborhood, the trends and the scatter of elemental abundance ratios are in good agreement with observations (see KN11 for the $[\mathrm{X} / \mathrm{Fe}]-[\mathrm{Fe} / \mathrm{H}]$ diagrams of the solar neighborhood, thick disk, and bulge). At the same $[\mathrm{Fe} / \mathrm{H}],[\mathrm{Mn} / \mathrm{Fe}]$ also shows an increasing trend with $[\mathrm{Fe} / \mathrm{H}]$ because $\mathrm{Mn}$ is more produced by SNe Ia than Fe. Odd-Z elements $(\mathrm{Na}, \mathrm{Al}$, and $\mathrm{Cu}$ ) show increasing trends at $[\mathrm{Fe} / \mathrm{H}] \lesssim-1$ because of the metallicity dependence of nucleosynthesis yields. In the bulge, the star formation timescale is so short that the $[\alpha / \mathrm{Fe}]$ plateau continues to $[\mathrm{Fe} / \mathrm{H}] \sim+0.3$. Because of the smaller contribution from SNe Ia, the majority of stars shows high $[\alpha / \mathrm{Fe}]$ and low $[\mathrm{Mn} / \mathrm{Fe}]$. $[(\mathrm{Na}, \mathrm{Al}, \mathrm{Cu}, \mathrm{Zn}) / \mathrm{Fe}]$ are also high because of the high metallicity in the bulge. The stellar population of the thick disk is neither disk-like nor bulge-like. For thick disk stars, $[\alpha / \mathrm{Fe}]$ is higher, and $[\mathrm{Mn} / \mathrm{Fe}]$ is lower than thin disk stars because of the short formation timescale. However, [( $\mathrm{Na}, \mathrm{Al}, \mathrm{Cu}, \mathrm{Zn}) / \mathrm{Fe}]$ are lower than bulge stars because of the lower chemical enrichment efficiency. This is because half of the thick disk stars have already formed in satellite galaxies before they accrete onto the disk, and the metals have been ejected from the satellite galaxies by galactic winds.

In the disk, metallicity radial and vertical gradients exist, but no $[\alpha / \mathrm{Fe}]$ radial gradient, which seems consistent with observations by SDSS and RAVE. These suggest that the chemical enrichment efficiency is higher in the center but the star formation timescale does not so much depend on the radius. In the bulge, metallicity and $[\alpha / \mathrm{Fe}]$ vertical gradients exist, which is caused by the increase of metal-rich and low $[\alpha / \mathrm{Fe}]$ populations at lower latitudes. For the thick disk stars, there is metallicity vertical, weak metallicity radial, and no $[\alpha / \mathrm{Fe}]$ radial gradients (see K14 for the radial profiles and histograms).

The time evolution of these gradients are important to determine the major physical process of the disk formation. In chemodynamical simulations like our model, metallicity radial gradients are steeper at higher redshifts (see KN11 for the figure). This time evolution is consistent with the observations of lensed disk galaxies at high redshifts (Pilkington et al. 2012). On the other hand, classical monolithic collapse models often 

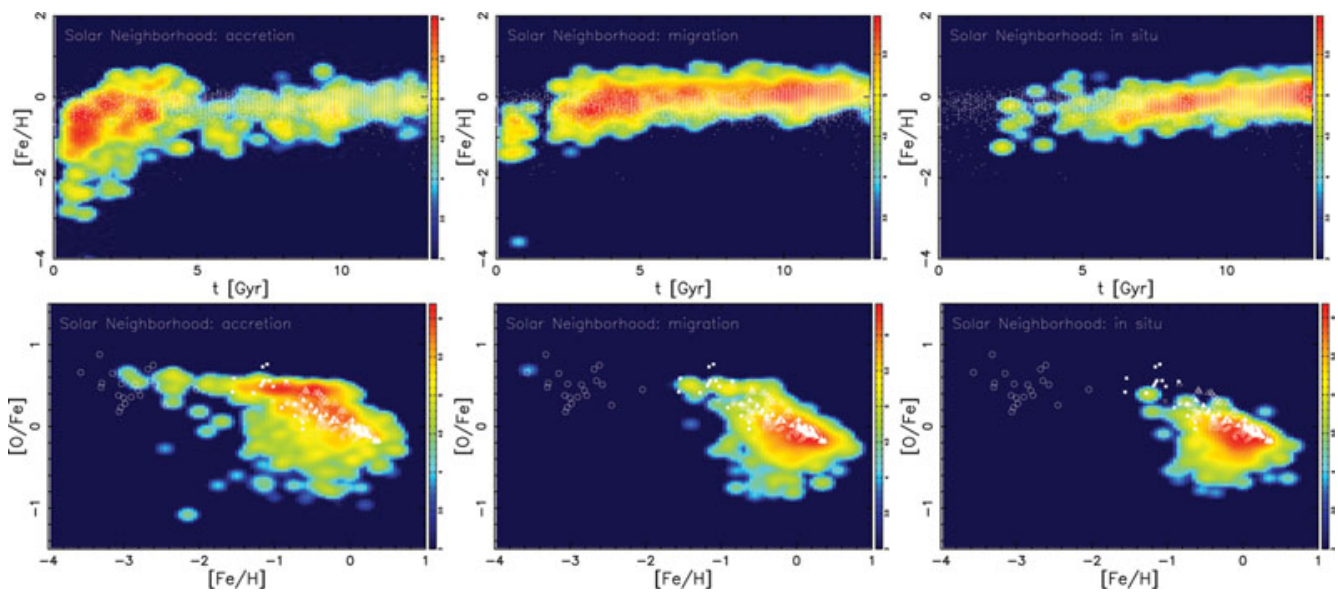

Figure 2. The age-metallicity and $[\mathrm{O} / \mathrm{Fe}]-[\mathrm{Fe} / \mathrm{H}]$ relations for stars that formed in-situ (right panels), and originate from migration (middle panels) and stellar accretion of mergers (left panels).

predict opposite evolution, and migration may have much faster evolution. Note that an inverse gradient is shown at $z \sim 3$ by Cresci et al. (2010), but there is no stable disk at such a high redshift in chemodynamical simulations from cosmological initial conditions, and this observation is not suitable for the discussion of Milky Way-size disks.

\section{Local inhomogeneous effect - the origin of the scatter in $[X / F e]$}

In this section, we discuss three processes that cause a scatter of stellar abundances. The first two processes are in the effect (IV), and the third process consists of the effects (I)-(III). By selecting stars from the locations when the stars formed, we show how each process affects on stellar populations in the solar neighborhood in Figure 2.

(1) Accretion of stars in merging satellites that have a different evolutionary history. Usually chemical enrichment efficiency is low because of the small potential well. No age-metallicity relation is expected. In Figure 2 , there are not many stars at $[\mathrm{Fe} / \mathrm{H}] \gtrsim 0$, and no time evolution. There is not very much contribution from SNe Ia. The most metal-poor population arise from this process.

(2) Migration of stars along the disk plane. Stars formed in the center can be located in the solar neighborhood at present. Because of the metallicity gradients, metallicity can be high. No age-metallicity relation is expected. In Figure $2,[\mathrm{Fe} / \mathrm{H}]$ is as high as $\sim 0$, and there is no time evolution. Most of these stars are disk stars and enriched by SNe Ia.

(3) There is an intrinsic scatter in the stars formed in the solar neighborhood because star formation and chemical enrichment depend on local density (in situ). In Figure 2, there is no old stars at $t \lesssim 2$ Gyr because the disk grow inside-out. The age-metallicity relation is clearly seen from $(t,[\mathrm{Fe} / \mathrm{H}])=(6 \mathrm{Gyr},-0.5)$ to $(13 \mathrm{Gyr},+0.5)$. The $[\mathrm{O} / \mathrm{Fe}]-[\mathrm{Fe} / \mathrm{H}]$ relation is seen from $[\mathrm{Fe} / \mathrm{H}] \sim-1$ to +0.5 . These are no metal-poor stars with $[\mathrm{Fe} / \mathrm{H}]$ $\lesssim-1.4$, which suggests that there is pre-enrichment from pre-existing supernovae. However, there is still a significant scatter in both relations. This is the local inhomogeneous enrichment effect.

In summary, the following phenomena occur in the case of inhomogeneous enrichment:

i) The age-metallicity relation is weak. In other words, the most metal-poor stars are not always the oldest stars. In our simulations, the metallicity of the first enriched stars 
reaches $[\mathrm{Fe} / \mathrm{H}] \sim-3$. At later times, star forming regions become denser, and both metal richer and poorer stars than $[\mathrm{Fe} / \mathrm{H}] \sim-3$ appear. ii) Some stars can be affected by SNe Ia at $[\mathrm{Fe} / \mathrm{H}] \lesssim-1$ even with our metallicity inhibition of SNe Ia. The SN Ia contribution is characterised by low $[\alpha / \mathrm{Fe}]$ and high $[\mathrm{Mn} / \mathrm{Fe}]$. iii) The scatter of elemental abundance ratios becomes large if the supernova yield depends on progenitor metallicity such as Na. iv) Some CEMP/NEMP stars can be explained with the local enrichment from AGB stars even without the binary effect. In fact, the observed $[\mathrm{N} / \mathrm{O}]-[\mathrm{O} / \mathrm{H}]$ trend can be reproduced with our simulation without including the effect of rotating massive stars (see K14 for the figure).

\section{The first chemical enrichment}

Despite more than ten years of surveys, no stars that have an elemental abundance pattern consistent with pair-instability supernovae (PISNe) have been found. The characteristics of PISN enrichment are: i) The odd-Z effect is much larger than $\sim 1$ dex. ii) $[(\mathrm{Si}, \mathrm{S}, \mathrm{Ar}, \mathrm{Ca}) / \mathrm{Fe}]$ are much larger than $[(\mathrm{O}, \mathrm{Mg}) / \mathrm{Fe}]$ because of more extensive explosive oxygen burning. iii) $[\mathrm{Cr} / \mathrm{Fe}]$ is much larger because of the larger incomplete Si-burning region. iv) $[(\mathrm{Co}, \mathrm{Zn}) / \mathrm{Fe}]$ are much smaller because of the much larger ratio between the complete and incomplete Si-burning regions. The star reported by Aoki et al. (2014) does not show the Si enhancement $([\mathrm{Si} / \mathrm{Mg}] \sim 0.1)$ and $[\mathrm{Co} / \mathrm{Fe}]$ is extremely low $(\sim-0.7)$, although the $[\mathrm{Mg} / \mathrm{Fe}]$ ratio is very low $(\sim-0.5)$. This abundance pattern may be explained with a anisotropic enrichment by jet-like explosions.

Instead, the elemental abundance patterns of extremely metal-poor (EMP) stars (Ishigaki et al. 2014, including the $[\mathrm{Fe} / \mathrm{H}]<-7$ star) and metal-poor damped Lyman $\alpha$ systems (Kobayashi et al. 2011c), in particular the large carbon enhancement, are well reproduced by faint supernovae. The central parts of supernova ejecta that contain most of the iron fall back onto the black hole, while the stellar envelopes that contain carbon are ejected as in normal supernovae. Therefore, the $[\mathrm{C} / \mathrm{Fe}]$ ratio of faint supernovae is as large as that of carbon-enhanced metal-poor (CEMP) stars. Among $\alpha$ elements, O and $\mathrm{Mg}$ are synthesized during hydrostatic burning and are located in the outskirts of ejecta. Therefore, faint supernovae often have high $[(\mathrm{O}, \mathrm{Mg}) / \mathrm{Fe}]$ ratios, depending on the mixing-fallback processes. The mixing-fallback effect is naturally expected in the case of hypernovae, which are jet-induced explosions (of rotating stars) followed by black hole formation. In supernovae, it is also possible that some degree of mixing occurs through Rayleigh-Taylor instability.

Some EMP stars show low $[\alpha / \mathrm{Fe}]$ ratios, which can be explained with low-mass $(\sim$ $13-15 M_{\odot}$ ) supernovae (Kobayashi et al. 2014). $\sim 10-20 M_{\odot}$ supernovae have a smaller mantle mass that contains $\alpha$ elements than more massive stars, and thus give lower $[\alpha / \mathrm{Fe}]$ ratios than the initial mass function (IMF) weighted values of core-collapse supernova yields, i.e., the plateau values of $[\alpha / \mathrm{Fe}]-[\mathrm{Fe} / \mathrm{H}]$ relations. These supernovae will leave a neutron star behind, and should be very common for the standard IMF weighted for the low-mass end. A couple of stars also show carbon enhancement, which is interesting. If there is a CEMP-low- $\alpha$ star with $[\mathrm{Zn} / \mathrm{Fe}] \sim[\mathrm{Co} / \mathrm{Fe}] \sim 0$, that would require a new population, i.e., $\sim 10-20 M_{\odot}$ faint supernovae that form black holes. For $\lesssim 20 M_{\odot}$ stars, they are believed to form neutron stars, and thus black-hole forming faint supernovae have not been discussed in previous works.

In summary, an intrinsic variation of $[\alpha / \mathrm{Fe}]$ ratios can be caused by the following enrichment sources: (1) SNe Ia, (2) low-mass supernovae, (3) hypernovae $\left(E / 10^{51} \mathrm{erg} \gtrsim 10\right.$ 

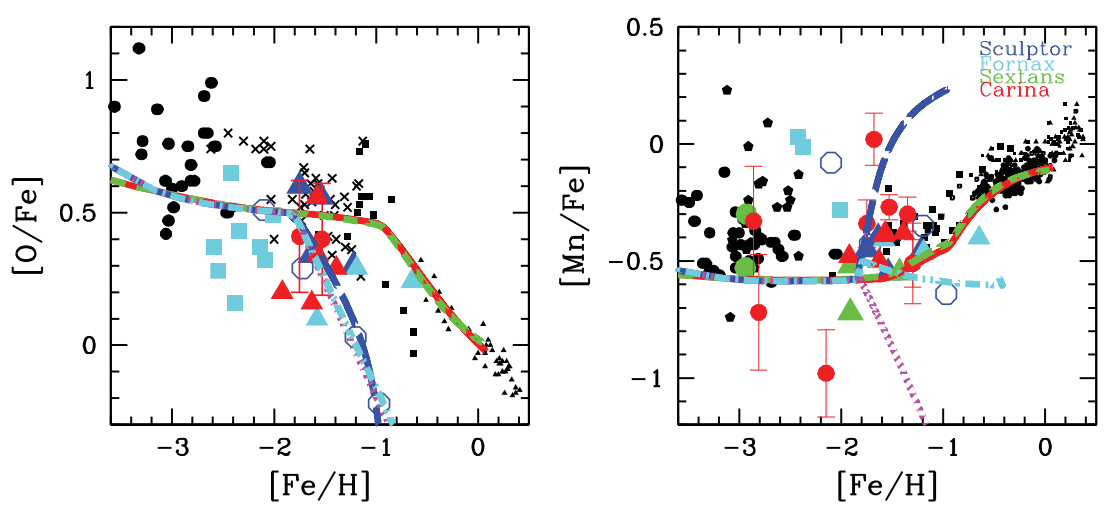

Figure 3. Evolution of elemental abundance ratios for the solar neighborhood with only normal SNe Ia (red solid lines) and a model with 50\% SNe Iax and 50\% sub-Ch SNe Ia (green shortdashed lines). The blue long-dashed, cyan dotted, and magenta dotted-dashed lines are for dSph galaxies with $100 \%$ SN Iax, $100 \%$ sub-Ch SNe Ia, and equal mix of the two, respectively. See Kobayashi et al. (2011, black) and Venn et al. (2012, color) for the observational data sources of the solar neighborhood and dSphs, respectively.

for $\gtrsim 25 M_{\odot}$, NKT13), (4) faint supernovae, and (5) PISNe. (1) and (2) are fully, (3) is partially, included in my chemodynamical simulations.

\section{Dwarf Spheroidal Galaxies}

The low $[\alpha / \mathrm{Fe}]$ ratios and their trends in dwarf spheroidal galaxies can be explained by subclasses of SNe Ia, such as SN 2002cx-like objects and sub-Chandrasekhar (Ch) mass explosions (Kobayashi et al. 2015), that have been discovered with recent extensive observations of supernovae. The $[\alpha / \mathrm{Fe}]$ ratios of stars in dSphs such as Fornax and Sagittarius are lower than those of the Galactic halo stars. This difference is often interpreted to indicate a larger contribution of SNe Ia in dSphs than that of the solar neighborhood. It is also possible to find a quite clear knee at lower metallicities; $[\mathrm{Fe} / \mathrm{H}] \sim-2$ in Sculptor, $\sim-1.5$ in Fornax, and so on (e.g., Tolstoy et al. 2009). If this knee is caused by SNe Ia, $[\mathrm{Mn} / \mathrm{Fe}]$ ratios should show an increasing trend with $[\mathrm{Fe} / \mathrm{H}]$ from this $[\alpha / \mathrm{Fe}]$ knee. However, in dSph galaxies, $[\mathrm{Mn} / \mathrm{Fe}]$ ratios are as low as in the Galactic halo stars for a wide range of metallicity (e.g., North et al. 2012). One possible scenario is the lack of contributions from massive core-collapse supernovae due to the incomplete sampling of IMF (e.g., Venn et al. 2012$)$. Less-massive supernovae $\left(\sim 20 M_{\odot}\right)$ give low $[\alpha / \mathrm{Fe}]$ ratios without changing $[\mathrm{Mn} / \mathrm{Fe}]$ ratios.

We propose another scenario. Figure 3 shows our new chemical evolution models that includes two possible channels in the single degenerate scenario: (1) double detonations in sub-Ch mass CO WDs, where a thin He envelope is developed with relatively low accretion rates after He novae even at low metallicities, and (2) carbon deflagrations in Ch-mass possibly hybrid $\mathrm{C}+\mathrm{O}+\mathrm{Ne}$ WDs, where WD winds occur at $[\mathrm{Fe} / \mathrm{H}] \sim-2.5$ at high accretion rates. These subclasses of SNe Ia are rarer than 'normal' SNe Ia and do not affect the chemical evolution in the solar neighborhood, but can be very important in metal-poor systems with stochastic star formation. In dwarf spheroidal galaxies in the Local Group, the decrease of $[\alpha / \mathrm{Fe}]$ ratios at $[\mathrm{Fe} / \mathrm{H}] \sim-2$ to -1.5 can be produced depending on the star formation history. SNe Iax give high [Mn/Fe], while sub-Ch-mass SNe Ia give low $[\mathrm{Mn} / \mathrm{Fe}]$, and thus a model including a mix of the two is favored by the available observations. 


\section{References}

Aoki, W, Tominaga, N., Beers, T. C., Honda, S., \& Lee, Y. S. 2014, Science, 345, 912

Cresci, G., Mannucci, F., Maiolino, R., et al. 2010, Nature, 467, 811

Ishigaki, M. N., Tominaga, N., Kobayashi, C., \& Nomoto, K. 2014, ApJ, 792, L32

Kobayashi, C. 2014, in IAU Symposium 298, 298, 167 (K14)

Kobayashi, C., Ishigaki, M. N., Tominaga, N., \& Nomoto, K. 2014, ApJ, 5, L5

Kobayashi, C., Karakas, I. A., \& Umeda, H. 2011a, MNRAS, 414, 3231

Kobayashi, C., Izutani, N., Karakas, A. I. et al. 2011b, ApJ, 739, L57

Kobayashi, C. \& Nakasato, N. 2011, ApJ, 729, 16 (KN11)

Kobayashi, C. \& Nomoto, K. 2009, ApJ, 707, 1466

Kobayashi, C., Nomoto, K., \& Hachisu, I. 2015, ApJ, 804, L24

Kobayashi, C., Springel, V., \& White, S. D. M. 2007, MNRAS, 376, 1465

Kobayashi, C., Tominaga, N., \& Nomoto, K. 2011c, ApJ, 730, L14

Kobayashi, C., Umeda, H., Nomoto, K., Tominaga, N., \& Ohkubo, T. 2006, ApJ, 653, 1145

Nomoto, K., Kobayashi, C., \& Tominaga, N. 2013, ARA\&A, 51, 457 (NKT13)

North, P., Cescutti, G., Jablonka, P., et al. 2012, A\&A, 541, 45

Pilkington, K., Few, C. G., Gibson, B. K., et al. 2012, A\&A, 540, A56

Tolstoy, E., Hill, V., \& Tosi, M. 2009, ARA\& $A$, 47, 371

Venn, K. A., Shetrone, M. D., Irwin, M. J., et al. 2012, ApJ, 751, 102 